\title{
Endobronchial Coil System versus Standard-of-Care Medical Management in the Treatment of Subjects with Severe Emphysema
}

\author{
Karin Klooster ${ }^{a}$ Arschang Valipour ${ }^{b} \quad$ Charles-Hugo Marquette ${ }^{c}$ Jacques Boutros ${ }^{c}$ Hervé Mal ${ }^{d}$ \\ Armelle Marceau ${ }^{d}$ Pallav L. Shah ${ }^{\mathrm{e}}$ Francesca Conway $^{\mathrm{e}}$ Gaëtan Deslée ${ }^{\mathrm{f}}$ Arnaud Bourding $^{\mathrm{g}}$ \\ Christophe Pison $^{\text {h }}$ Christian Grah ${ }^{i}$ Martin Hetzel ${ }^{j} \quad$ Christian Schumann $^{k}$ Romain Kessler ${ }^{1}$ \\ Ralf-Harto Huebner ${ }^{m}$ Dirk Skowasch ${ }^{n} \quad$ Kaid Darwiche $^{\circ}$ Peter Hammerl $^{\mathrm{p}}$ Franz Stanzel $^{\mathrm{q}}$ \\ Michaela Bezzi ${ }^{r}$ Hervé Dutau ${ }^{s}$ Felix J.F. Herth ${ }^{t}$ Dirk-Jan Slebos ${ }^{a}$

\begin{abstract}
aDepartment of Pulmonary Diseases, University of Groningen, University Medical Center Groningen, Groningen, The Netherlands; bKarl-Landsteiner-Institute for Lung Research and Pulmonary Oncology, Klinik Floridsdorf, Floridsdorf, Austria; 'Université Côte d'Azur, FHU OncoAge, CHU de Nice, Nice, France; 'Hôpital Bichat - Claude Bernard, Paris, France; eDepartment of Respiratory Medicine, Royal Brompton Hospital, London, United Kingdom; ${ }^{\mathrm{f}} \mathrm{CHU}$ de Reims, Reims, France; ${ }^{9} \mathrm{CHU}$ de Montpellier, Montpellier, France; ${ }^{\mathrm{h}} \mathrm{CHU}$ de Grenoble, Grenoble, France; 'Gemeinschaftskrankenhaus Havelhöhe

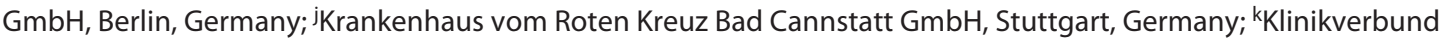
Allgäu, Immenstadt, Immenstadt, Germany; 'Nouvel Hôpital Civil, Strasbourg, France; ${ }^{\mathrm{m} C h a r i t e ́ ~ B e r l i n, ~ B e r l i n, ~ G e r m a n y ; ~}$ nUniversitätsklinkum Bonn, Bonn, Germany; ${ }^{\circ}$ Department for Interventional Pneumology, Ruhrlandklinik - University

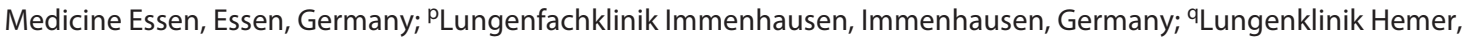

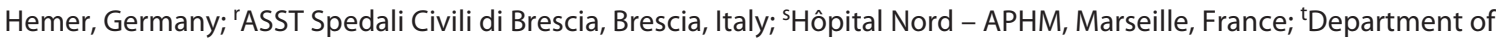
Internal Medicine, Pulmonary and Critical Care Medicine Thoraxklinik and Translational Lung Research Center Heidelberg University Heidelberg, Heidelberg, Germany
\end{abstract}

\section{Keywords}

Emphysema · Bronchoscopic lung volume reduction · Endobronchial coil treatment $\cdot$ Hyperinflation

\begin{abstract}
Background: Bronchoscopic lung volume reduction using endobronchial coils is a new treatment for patients with severe emphysema. To date, the benefits have been modest and have been suggested to be much larger in patients with severe hyperinflation and nonmulti-comorbidity. Objective: We aimed to evaluate the efficacy and safety of endobronchial coil treatment in a randomized multicenter clinical trial using optimized patient selection. Method: Patients with severe emphysema on HRCT scan with severe hyperinflation
\end{abstract}

(residual volume $[R V] \geq 200 \%$ predicted and RV/total lung capacity $[\mathrm{TLC}]>55 \%$ ) were randomized to coil treatment or control. Primary outcome measures were differences in the forced expiratory volume in $1 \mathrm{~s}\left(\mathrm{FEV}_{1}\right)$ and St George's Respiratory Questionnaire (SGRQ) total score at 6 months. Results: Due to premature study termination, a total of 120 patients (age $63 \pm 7$ years, $\mathrm{FEV}_{1} 29 \pm 7 \%$ predicted, RV $251 \pm 41 \%$ predicted, RV/TLC $67 \pm 6 \%$, and SGRQ $58 \pm 13$ points), instead of 210 patients, were randomized. At study termination, 91 patients (57 coil and 34 control) had 6-month results available. Analyses showed significantly greater improvements in favor of the coil group. The increase in $\mathrm{FEV}_{1}$ was greater in the coil group than that in the control group by $+10.3[+4.7$ to $+16.0] \%$ and in SGRQ by $-10.6[-15.9$ to -5.4$]$ points. At study termination, there were $5(6.8 \%)$ deaths in the coil co- karger@karger.com www.karger.com/res

Karger $\stackrel{\text { ' }}{5}$

BOPEN ACCESS
(C) 2021 The Author(s)

Published by S. Karger AG, Basel

This is an Open Access article licensed under the Creative Commons Attribution-NonCommercial-4.0 International License (CC BY-NC) (http://www.karger.com/Services/OpenAccessLicense), applicable to the online version of the article only. Usage and distribution for commercial purposes requires written permission.
Correspondence to:

Karin Klooster, k.klooster@umcg.nl 
hort reported. Conclusion: Despite early study termination, coil treatment compared to control results in a significant improvement in the lung function and quality of life benefits for up to 6 months in patients with emphysema and severe hyperinflation. These improvements were of clinical importance but were associated with a higher likelihood of serious adverse events.

(c) 2021 The Author(s)

Published by S. Karger AG, Basel

\section{Introduction}

In the past 10 years, bronchoscopic lung volume reduction using nitinol endobronchial coils has emerged as a new treatment for COPD patients with severe emphysema. Endobronchial coil treatment has been shown to improve the quality of life, exercise capacity, and pulmonary function in severe emphysema patients, with an acceptable safety profile [1-6].

Post hoc analysis from the RENEW trial [2], an international, multicenter, randomized controlled trial assessing endobronchial coil treatment in emphysema patients with severe lung hyperinflation, identified baseline predictors that identified patients who most likely significantly benefitted from endobronchial coil treatment. This post hoc analysis showed that both significant hyperinflation and quantitative CT scan analysis are critical for patient selection and treatment planning for endobronchial coil therapy. CT scan analysis is important to identify optimal lobar treatment and to exclude patients with insufficient emphysema, whereas visual assessment identifies patients with signs of airway disease associated with worse outcomes [7]. These findings need prospective confirmation to establish the profile of patients with severe emphysema who should be offered the endobronchial coil treatment.

On the basis of these findings and previous trial results, the ELEVATE randomized clinical trial was designed to assess 6-month effectiveness and safety of coil treatment using this optimized patient selection. Based on these promising findings, the ELEVATE study was initiated. It was a prospective, multicenter, open-label, randomized controlled study. The ELEVATE study was conducted to assess 6-month effectiveness and safety of endobronchial coils on the quality of life and lung function in patients with severe lung hyperinflation and advanced emphysema. The study was terminated prematurely by the study sponsor (Boston Scientific Corporation [BSC], USA), for business-related reasons after $57 \%$ of the planned patients were randomized.

Endobronchial Coil Treatment for the

Treatment of Emphysema

\section{Methods}

\section{Study Population}

Severe emphysema patients meeting the indications for use per the approved CE marked instructions for use for the coil system treatment were included. And, in addition, the study population included all subjects who have met the inclusion criteria. The main eligibility criteria included COPD patients with severe emphysema, with a $15 \%$ predicted $\leq$ post-bronchodilator forced expiratory volume in $1 \mathrm{~s}\left(\mathrm{FEV}_{1}\right) \leq 45 \%$ predicted, total lung capacity $>100 \%$ predicted, and residual volume (RV) $\geq 200 \%$ predicted. Patients with predominant small airways disease defined as significant bronchiectasis with sputum production completion were excluded from enrollment. All inclusion and exclusion criteria for this study are listed in the "Protocol of a Randomized Controlled Study of the PneumRx Endobronchial Coil System versus Standard-of-Care Medical Management in the Treatment of Subjects with Severe Emphysema" [8].

\section{Study Design}

This was a prospective, multicenter, open-label, randomized $(2: 1)$ controlled study comparing outcomes in subjects treated with the coil system (the coil treatment group) to a medically managed control group (the usual care group). The follow-up duration was planned for 24 and 36 months (in France) post-initial treatment. The primary endpoint was measured at 6 months. Enrollment of 210 patients was planned, and patients were randomized in a 2:1 ratio of coil treatment to usual care, respectively (140 coil treatment group patients and 70 usual care group patients), across up to 30 sites. Randomization was stratified by site and heterogeneity of emphysema.

\section{Study Procedure}

Procedural planning was performed in accordance with the instruction for use. The coil system was a two-part system that consists of sterile implants ( 3 sizes of coils: 100,125 , and $150 \mathrm{~mm}$ ) and sterile, disposable, single-use delivery system consisting of a cartridge, catheter, guidewire, and forceps. The coil was delivered through a standard 2.8-mm-inner diameter therapeutic bronchoscope. Each coil implantation procedure must be performed under fluoroscopy. The use of general anesthesia or conscious sedation was at the discretion of the bronchoscopist and anesthesiologist. Coil treatment was bilaterally performed with procedures separated by 1-3 months, with the most affected lobe of the lung being treated on each side. Ideally, 10-12 coils were placed in an upper lobe and 12-14 in a lower lobe. Identification of the most affected lobe was by densitometry (quantitative CT analysis provided by PneumRx QCT service) of a high-resolution CT of the lungs.

\section{Study Measurements}

At baseline and 6-month follow-up, the pulmonary function test, 6-minute walk test, and inspiratory and expiratory HRCT were performed and the quality-of-life assessments were completed.

\section{Effectiveness Outcomes}

The co-primary endpoints are the percent change in $\mathrm{FEV}_{1}$ and change in St George's Respiratory Questionnaire (SGRQ) total score from baseline to 6 months, with a difference of $10 \%$ in percent change in $\mathrm{FEV}_{1}$ and 7 points in SGRQ between coil treatment and usual care groups. 


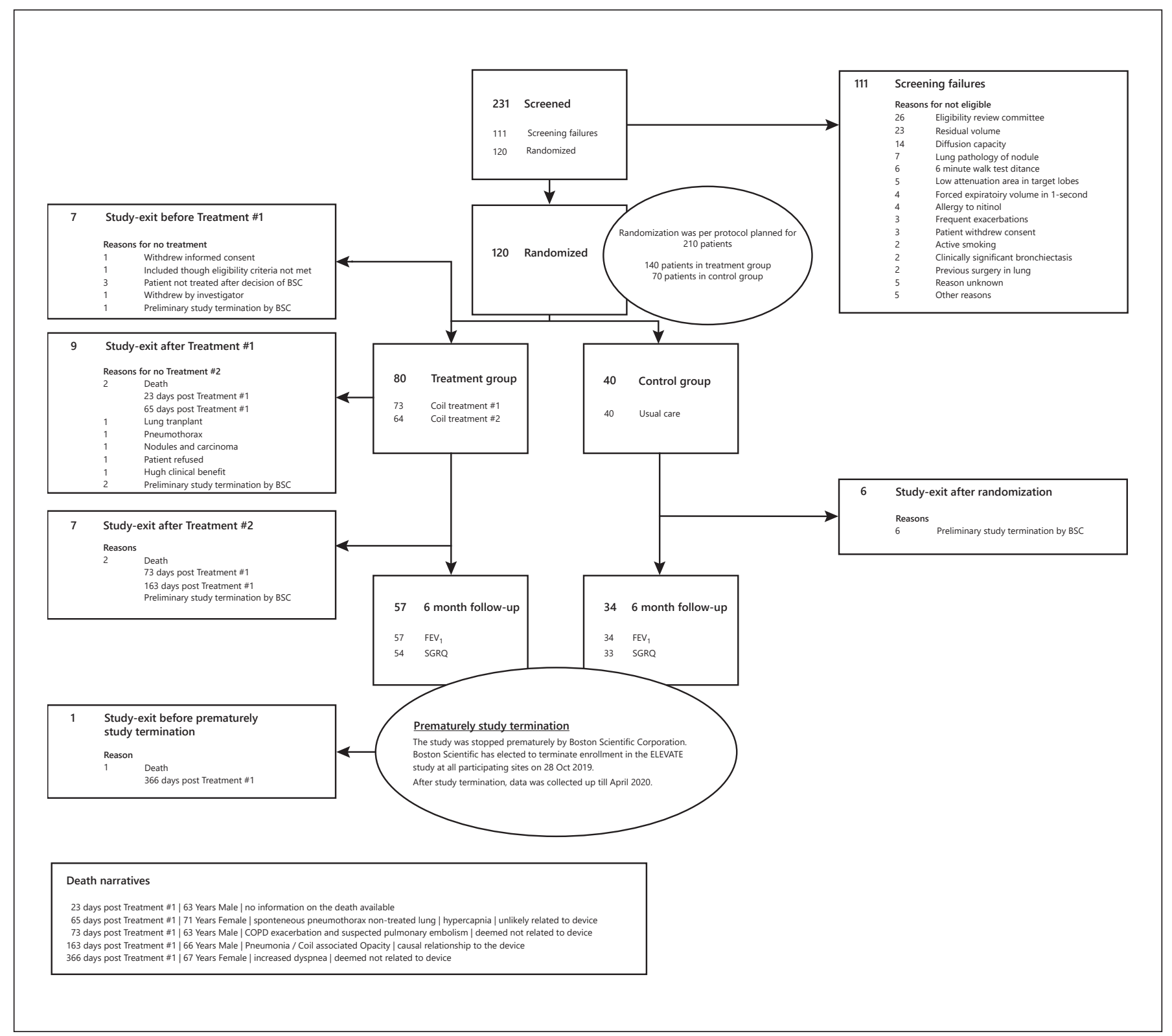

Fig. 1. Patient flow. BSC, Boston Scientific Corporation; $\mathrm{FEV}_{1}$, forced expiratory volume $1 \mathrm{~s}$; SGRQ, St George's Respiratory Questionnaire.

\section{Statistical Analysis}

Data reported were based on the available data of the frozen trial database from April 6, 2020. Efficacy analyses were performed with the available data. $\mathrm{FEV}_{1}$ outcomes were not normally distributed. Data are presented as median values (95\% confidence interval [CI]). The Wilcoxon signed-rank test was used to calculate the change from baseline at 6 months in $\mathrm{FEV}_{1}$. The Hodges-Lehmann estimator was used to calculate the median of the group difference (95\% CI). The Mann-Whitney U test was used to calculate the median difference in change from baseline between groups for $\mathrm{FEV}_{1}$. SGRQ outcomes are normally distributed. Data are presented as mean values (95\% CI). The paired $t$-test was used to calculate the change from baseline at 6 months in SGRQ. The independent samples $t$-test was used to calculate the mean difference in change from baseline between groups for SGRQ. $p$ values $<0.05$ were considered significant. The change from baseline at 6 months in $\%$ for $\mathrm{FEV}_{1}$ and in points for SGRQ for each individual patient is displayed per group. Minimal clinical important differences (MCIDs) were used to calculate the proportion of responders. The MCID for FEV $\geq 10 \%$ change [9] and MCID for SGRQ $\geq-7$ points change [10] were used. Safety is reported descriptively. Reported incidence rates through 6 months are summarized for treatment and control groups. All analyses were performed with IBM SPSS statistics version 23 . 
Table 1. Baseline demographic characteristics of randomized patients

\begin{tabular}{lcc}
\hline Characteristic & $\begin{array}{l}\text { Coil treatment } \\
(N=80)\end{array}$ & $\begin{array}{l}\text { Usual care } \\
(N=40)\end{array}$ \\
\hline Demographic & & \\
Age, years & $64[44-84]$ & $63[52-79]$ \\
Female, $n(\%)$ & $41(51 \%)$ & $21(53 \%)$ \\
BMI, kg/m ${ }^{2}$ & $23[15-30]$ & $24[18-30]$ \\
Smoking history, pack-years & $43[7-100]$ & $40[10-133]$ \\
Lung function & & \\
FVC, & $2.43[1.20-4.53]$ & $2.51[1.58-4.28]$ \\
FEV, L & $0.71[0.45-1.45]$ & $0.71[0.41-1.40]$ \\
FEV,$\%$ predicted & $29[16-46]$ & $28[17-41]$ \\
FEV 1 /FVC, \% & $31[18-53]$ & $28[19-46]$ \\
RV, L & $5.30[3.66-8.84]$ & $5.30[3.80-7.77]$ \\
RV, \% predicted & $243[199-369]$ & $241[200-371]$ \\
RV/TLC & $67[55-83]$ & $66[54-78]$ \\
SGRQ, points & $56[41-90]$ & $55[19-86]$ \\
\hline
\end{tabular}

Data are presented as median [minimum-maximum] or $n(\%)$, unless otherwise stated. $\mathrm{FEV}_{1}$, forced expiratory volume in $1 \mathrm{~s} ; \%$ predicted; FVC, forced vital capacity; RV, residual volume; TLC, total lung capacity; SGRQ, St George's Respiratory Questionnaire. Baseline characteristics were similar in both groups.

\section{Results}

\section{Premature Study Termination}

This study was initially sponsored by PneumRx/BTG, CA, USA. On August 19, 2019, BTG was acquired by BSC, USA. On October 28, 2019, BSC has elected to discontinue the product pipeline of the ELEVAIR ${ }^{\mathrm{TM}}$ endobronchial coil system, resulting in premature study termination. BSC's decision was inconsiderate with the wish of all participating investigators to continue this unique therapy and was not driven by study results. After study termination, coordinators were able to enter data into the data entry system up till April 2020. Data for this manuscript were based on a snapshot of the database from April 6, 2020.

\section{Patients}

Study enrollment began in May 2018. Enrollment was prematurely capped at 120 patients (57\%), and the last patient was treated on October 28, 2019. There were a total of 80 patients randomized to the treatment group and 40 patients randomized to the control group at 20 European sites. By 6 months, efficacy results were available from 91 COPD patients (57 coil treatment and 34 con- trol). Figure 1 shows the patient flow in the ELEVATE study and Table 1 the demographic characteristics of the randomized patients.

\section{Treatment Details}

The first coil treatment was performed in 73 patients (91\% of the 80 patients who were randomized for the coil treatment group). A total of 64 patients were bilaterally treated. A mean of 11.2 coils were used in the first treatment, and a mean of 10.1 coils were used in the second treatment. The mean procedure time of the first treatment was $44.85 \mathrm{~min}$, and the mean procedure time of the second treatment was $41.60 \mathrm{~min}$.

\section{Efficacy Outcomes}

Based on the available data, at 6 months, the coil group $(N=57)$ showed significant improvements for the co-primary endpoints ( $\mathrm{FEV}_{1}$ and SGRQ) compared to control $(N=34)$, and a significantly higher response rate for these outcomes in favor of the patients in the coil treatment group (Fig. 2). There was also improvement in the severity of hyperinflation. The betweengroup difference in $\mathrm{RV}$ was $-460 \mathrm{~mL}$ ( -716 to $-203 \mathrm{~mL}$; $p=0.001)$ in favor of patients in the coil treatment group. The proportion of patients achieving an MCID of $>-350 \mathrm{~mL}$ in $\mathrm{RV}$ [11] was for patients in the coil treatment group 50 versus $23 \%$ for patients in the control group $(p=0.007)$.

\section{Safety}

At database closure, there were $5(6.8 \%)$ deaths in the coil group and $0(0 \%)$ deaths in the control group reported. All deaths occurred posttreatment. Death narratives: 23 days posttreatment\# 1 (63-year-old man), no information on the death available; 65 days posttreatment\#1 (71-year-old woman), spontaneous pneumothorax nontreated lung and hypercapnia, unlikely related to device; 73 days posttreatment\# 1 (63-year-old man), COPD exacerbation and suspected pulmonary embolism, deemed not related to device; 163 days posttreatment\#1 (66-yearold man), pneumonia with coil-associated opacity, causal relationship to the device; and 366 days posttreatment\#1 (67-year-old woman) increased dyspnea, deemed not related to device. In the coil group, 30 patients (41.1\%) experienced at least one serious adverse event (SAE), with a total of 45 SAEs reported up till database closure (most frequently: pneumonia $11.0 \%$, COPD exacerbation $4.1 \%$, and pneumothorax $4.1 \%$ ). In the control group, 3 patients (7.5\%) experienced at least one SAE (all COPD exacerbation). 


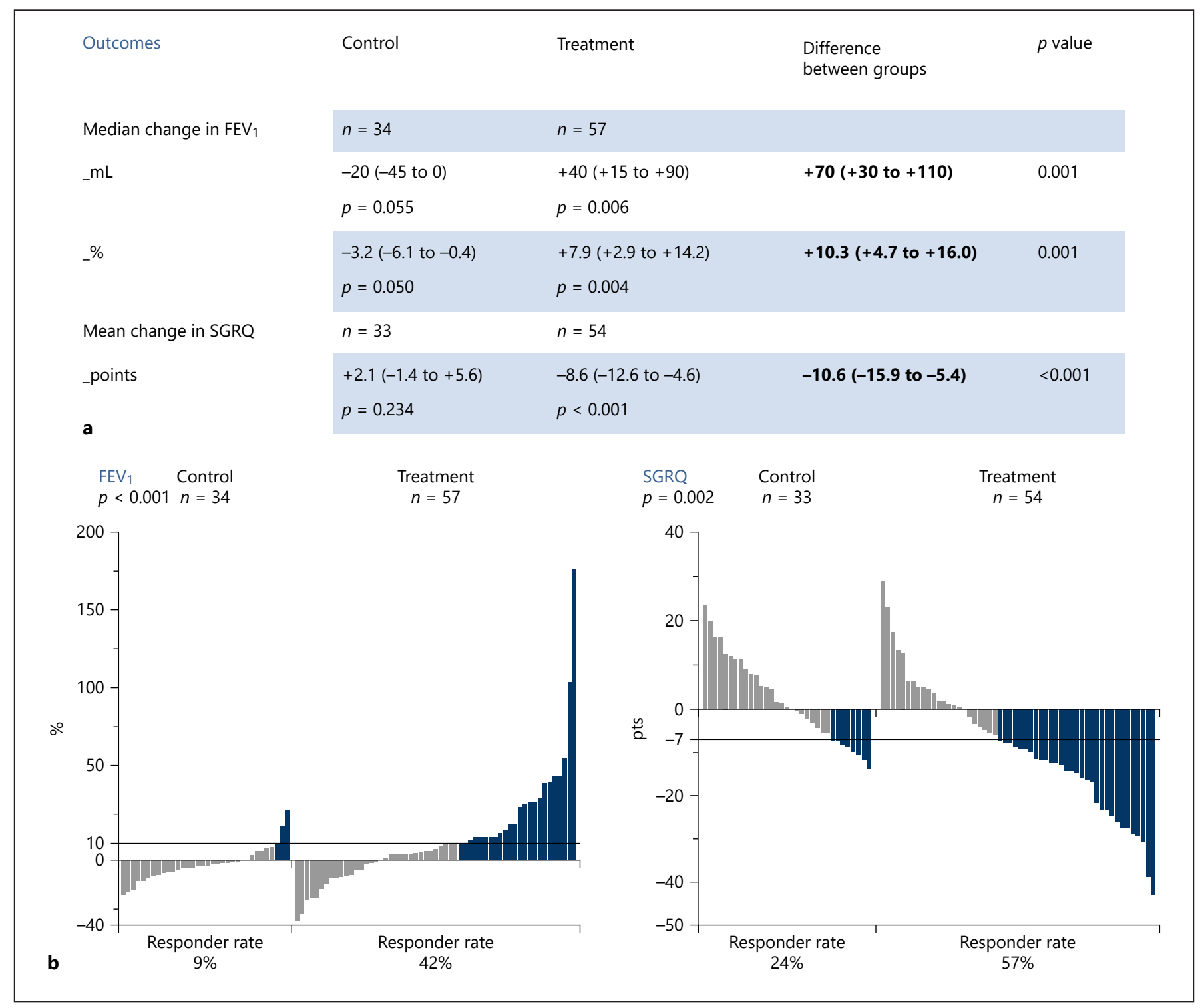

Fig. 2. Efficacy outcomes. Data reported are based on the available data of the frozen trial database from April 6, 2020. Efficacy and responder analyses were performed with the available data. a Primary endpoints at 6-month follow-up. $\mathrm{FEV}_{1}$ outcomes are not normally distributed and are presented as median values (95\% CI). SGRQ outcomes are normally distributed and are presented as mean values $(95 \% \mathrm{CI})$. b MCID responder results. The change from baseline at 6 months in $\%$ for $\mathrm{FEV}_{1}$ and in points for SGRQ

\section{Discussion}

Despite early study termination, the endobronchial coil treatment compared to control results in an improvement in the lung function and quality-of-life benefits for up to 6 months in the selected COPD patients with em- for each individual patient is displayed per group. MCID for $\mathrm{FEV}_{1}$ is $\geq 10 \%$ change and MCID for SGRQ is $\geq-7$ points change is a responder. One bar represents one patient. Light gray-colored bars represent patients not reaching the MCID. Blue colored bars represent patients who did reach the MCID. $\mathrm{FEV}_{1}$, forced expiratory volume $1 \mathrm{~s}$; MCID, minimal clinical important difference; SGRQ, St George's Respiratory Questionnaire; CI, confidence interval.

physema and severe hyperinflation and absence of predominant small airways disease and/or significant bronchiectasis. The improvements in both $\mathrm{FEV}_{1}$ and quality of life were of clinical importance but were associated with a higher likelihood of SAEs. These outcomes confirm the previously published findings using this treatment [1-4]. 
Our analysis was limited due to the premature study termination, resulting in a limited and incomplete data set. Instead of 210 patients randomized, there were 120 (57\%) patients randomized. Since the database was locked, analysis could only be performed on the data which were entered into the electronic database system before April 6, 2020, resulting in 6-month follow-up efficacy data from 91 patients. Also, the follow-up period of 2 years after treatment\#1 was not completed.

There was a higher mortality in the coil group than the standard of care (6.8 vs. 0\%). No definitive results could be presented due to incomplete follow-up. Compared to previous trials investigating the coil treatment, the percentage of death seems to be similar in the treatment group. In the RENEW trial [2], the percentage of death was 6.5 versus $5.1 \%$ in the control arm, and in the REVOLENS [1], it was 8 versus $6 \%$. However, in this dataset, the percentage of deaths in the control group was very low; this is probably due to the incomplete dataset and follow-up.

To date, only lung volume reduction surgery and bronchoscopic lung volume reduction using one-way valves reached the evidence level to be used outside of clinical trials [12]. Since only a small percentage (10-20\%) of our total referred severe emphysema patients is eligible for these more established advanced treatments [13], an additional lung volume reduction treatment, such as this coil treatment, is highly necessary to treat our suffering patients who hardly have any alternative treatments [12-14].

\section{Conclusion}

Despite early study termination, coil treatment compared to control results in an improvement in the lung function and quality-of-life benefits for up to 6 months in patients with emphysema and severe hyperinflation. The investigators collaboratively believed that publishing the available results could promote future developments of similar worthwhile techniques (NCT04520152) for our severe COPD patients with emphysema, who hardly have any alternative treatments, particularly in the presence of incomplete interlobar fissures and/or a more homogeneous emphysema distribution.

\section{Acknowledgements}

The authors would like to thank the voluntary contribution of the patients that participated in the ELEVATE study. They would like to thanks the following investigators who where initiated for the study: Dr. med. Daniel Drömann (Universitätsklinikum
Schleswig-Holstein, Germany), Prof. Dr. med. Susanne Lang (Waldklinikum Gera, Germany), PD Dr. med. Hans Klose (Universitätsklinikum Hamburg-Eppendorf, Germany), PD. Dr. Daniel Franzen (University Hospital Zurich, Switzerland), and Dr. Samy Lachkar (CHU de Rouen, France). They would also like to thank Ashley Burns-Keefe and Joerg Rennecke for their involvement and cooperation in this study.

\section{Statement of Ethics}

The study protocol was approved by all participating sites' local medical Ethics Committees. All patients provided informed consent before screening measurements were performed. The trial was registered under clinicaltrials.gov (identifier NCT03360396).

\section{Conflict of Interest Statement}

Karin Klooster, PhD: travel reimbursement and speakers fee from PulmonX, outside of the submitted work. Arschang Valipour, MD, PhD: nothing to disclose. Charles-Hugo Marquette, $\mathrm{MD}, \mathrm{PhD}$ : nothing to disclose. Jacques Boutros, MD: nothing to disclose. Hervé Mal, MD, PhD: nothing to disclose. Armelle Marceau, MD, PhD: nothing to disclose. Pallav L. Shah, MD, FRCP: personal fees from PneumRX/BTG as consultant on the scientific advisory board, other: sponsorship to Imperial College for a bronchoscopy course from ERBE, Cook medical, Medtronic, Boston Scientific, Broncus, PulmonX, Olympus, and PneumRX/BTG, outside of the submitted work. Francesca Conway, MBBS: nothing to disclose. Gaëtan Deslée, MD, PhD: personal fees from BTG/PneumRx, during the conduct of the study; personal fees from Nuvaira, AstraZeneca, Bohringer, Chiesi, and Novartis, outside of the submitted work. Arnaud Bourdin, MD, $\mathrm{PhD}$ : grants, personal fees, nonfinancial support, and other from GSK, Astra Zeneca, Boeringher Ingelheim, Novartis, Chiesi Farma, Teva, Sanofi Regeneron, and Actelion/Jansen, other from United Therapeutics, other from Pulsar, personal fees, nonfinancial support and other from Roche, from null, outside of the submitted work. Prof. Christophe Pison, MD, PhD: PneumRx financed the CHUGA to conduct this trial. Dr. Christian Grah, MD, PhD: nothing to disclose. Prof. Dr. Martin Hetzel, $\mathrm{MD}, \mathrm{PhD}$ : personal fees from BTG, during the conduct of the study. Prof. Dr. Christian Schumann, MD, PhD: nothing to disclose. Prof. Romain Kessler, MD, PhD: grants from Pneum$\mathrm{Rx}$, during the conduct of the study. Priv.-Doz Dr. Ralf-Harto Hübner, MD: personal fees from PulmonX, personal fees from Olympus, outside of the submitted work. Prof. Dr. Dirk Skowasch, MD, PhD: nothing to disclose. Priv.-Doz. Dr. Kaid Darwiche, MD, PhD: grants from Boston Scientific, outside of the submitted work. Dr. Peter Hammerl, MD, PhD: nothing to disclose. Dr. Franz Stanzel, MD, PhD: nothing to disclose. Dr. Michaela Bezzi, MD, PhD: nothing to disclose. Dr. Hervé Dutau, $\mathrm{MD}, \mathrm{PhD}$ : nothing to disclose. Felix J.F. Herth, MD, PhD: treated patients in clinical trials, sponsored by PneumRx/BTG, USA, PulmonX, Switzerland, and Spiration, USA. Advisor of supporting companies. Dirk-Jan Slebos, MD, PhD: grants and nonfinancial support from PneumRx/BTG, during the conduct of the study; grants, nonfinancial support, and other from Nuvaira, 
USA; grants, personal fees, nonfinancial support, and other from PulmonX, USA; and grants, nonfinancial support, and other from CSA Medical, USA, outside of the submitted work.

\section{Funding Sources}

The ELEVATE study was sponsored by BTG plc, PneumRx, Inc. of Santa Clara, CA, USA. BTG plc was acquired by Boston Scientific Corporation (BSC) on August 19, 2019.

\section{Author Contributions}

K.K. and D.J.S. performed data analyses and wrote the first version of the manuscript. All authors contributed to acquisition of data and reviewed and approved the manuscript.

\section{References}

1 Deslée G, Mal H, Dutau H, Bourdin A, Vergnon JM, Pison C, et al. Lung volume reduction coil treatment vs usual care in patients with severe emphysema: the REVOLENS randomized clinical trial. JAMA. 2016;315(2): $175-84$.

2 Sciurba FC, Criner GJ, Strange C, Shah PL, Michaud G, Connolly TA, et al. Effect of endobronchial coils vs usual care on exercise tolerance in patients with severe emphysema: the renew randomized clinical trial. JAMA. 2016;315(20):2178-89.

3 Slebos DJ, Hartman JE, Klooster K, Blaas S, Deslee G, Gesierich W, et al. Bronchoscopic coil treatment for patients with severe emphysema: a meta-analysis. Respiration. 2015; 90(2):136-45.

4 Shah PL, Zoumot Z, Singh S, Bicknell SR, Ross ET, Quiring J, et al. Endobronchial coils for the treatment of severe emphysema with hyperinflation (RESET): a randomised controlled trial. Lancet Respir Med. 2013;1(3): 233-40.
5 Hartman JE, Shah PL, Sciurba F, Herth FJF, Slebos DJ. Endobronchial coils for emphysema: dual mechanism of action on lobar residual volume reduction. Respirology. 2020; 25(11):1160-6.

6 Klooster K, Ten Hacken NH, Franz I, Kerstjens HA, Van Rikxoort EM, Slebos DJ. Lung volume reduction coil treatment in chronic obstructive pulmonary disease patients with homogeneous emphysema: a prospective feasibility trial. Respiration. 2014;88(2):116-25.

7 Slebos DJ, Cicenia J, Sciurba FC, Criner GJ, Hartman JE, Garner J, et al. Predictors of response to endobronchial coil therapy in patients with advanced emphysema. Chest. 2019;155(5):928-37.

8 Herth FJF, Slebos DJ, Shah PL, Hetzel M, Schmid-Bindert G, Laprad AS, et al. Protocol of a randomized controlled study of the PneumRx endobronchial coil system versus standard-of-care medical management in the treatment of subjects with severe emphysema (ELEVATE). Respiration. 2019;98:512-20.

9 Donohue JF. Minimal clinically important differences in COPD lung function. COPD. 2005;2(1):111-24.
10 Welling JBA, Hartman JE, Ten Hacken NHT, Klooster K, Slebos DJ. The minimal important difference for the St George's Respiratory Questionnaire in patients with severe COPD. Eur Respir J. 2015;46(6):1598-604.

11 Hartman JE, Ten Hacken NH, Klooster K, Boezen HM, De Greef MH, Slebos DJ. The minimal important difference for residual volume in patients with severe emphysema. Eur Respir J. 2012;40(5):1137-41.

12 Herth FJF, Slebos DJ, Criner GJ, Valipour A, Sciurba F, Shah PL. Endoscopic lung volume reduction: an expert panel recommendation: update 2019. Respiration. 2019;97(6):548-57.

13 Welling JB, Hartman JE, Augustijn SW, Kerstjens HA, Vanfleteren LE, Klooster K, et al. Patient selection for bronchoscopic lung volume reduction. COPD. 2020;15:871-81.

14 Pietzsch JB, Busca R, Rott C, Geisler BP, Weber SA, Slebos DJ, et al. Adoption patterns of bronchoscopic lung volume reduction procedures in Germany and predicted procedure volumes for other European countries. Respiration. 2018;97(1):34-41. 